\title{
475174 - AWAKE LAPAROSCOPY FOR PERITONEAL DIALYSIS CATHETER INSERTION
}

\author{
Arun Prasad, MBBS, DA, FRCA, Lakshmikumar Venkat Raghavan, MD FRCA, \\ Gopakumar Nair, MD \\ Anesthesia, Toronto Western Hospital, Toronto, ON, Canada
}

Introduction: Patients with end stage renal failure needing Peritoneal Dialysis (PD) often undergo insertion of PD catheter under local anesthesia. This method of blind insertion runs the risk of misplacement, early blockage and need for repeat procedures1. Laparoscopic insertion improves the success of PD cannula placement but this method has been limited by the need for general anesthesia. Aim of our study was to review the anesthetic management of patients undergoing laparoscopic PD cannula insertion under conscious sedation.

Methods: After ethics board approval, we retrospectively reviewed all patients who had PD catheter insertion done with Nitrous Oxide pneumoperitonium under local anesthetic infiltration and conscious sedation at our institution over the period February 2006 to January 2007. The data collected included patient demographics, coexisting medical conditions, anesthetic drugs used, perioperative hemodynamics, post-operative pain scores and complications.

Results: Totally, 40 patients underwent the procedure during this time period of which 35 were day cases. The mean age was 61.9 years (with a range of 40-96), 17 of them were male, and mean BMI was $25.77 \mathrm{~kg} / \mathrm{m} 2$ (range: 15.8 to 39), The average duration of the procedure was 57 minutes (range: 30 -105). All patients were given conscious sedation except two that needed GA-one for patient request and one due to perioperative discomfort. The drugs used for sedation included midazolam (100\%), fentanyl (75\%), propofol (70\%), remifentanil (20\%) and alfentanil (7.5\%). The most common combination was midazolam, fentanyl and propofol, followed by midazolam, and fentanyl. The peroperative events and post-operative complications are summarized in table 1. No major complication or unanticipated admission had occurred in this group. None of the patients were readmitted for catheter reinsertion.

Discussion: In our experience, conscious sedation for day case laparoscopic PD catheter insertion has been a successful and valuable alternative to GA in this group of high-risk patients

References: 1. Surg Endosc. 2000 Mar; 14(3): 308-9; 2. J Am Coll Surg. 2002 Aug; 195(2): 173-9; discussion 179-80.

Complications 


\begin{tabular}{|c|c|}
\hline Perioperative events & Number (n) of patients (\%) \\
\hline Hemodynamic instability $( \pm 20 \%$ of systolic BP & $12(30 \%)$ \\
\hline Peroperative nausea \& retching & $5(12.5 \%)$ \\
\hline Conversion to GA & $1(2.5 \%)$ \\
\hline Postoperative pain score $\geq 4$ (moderate to severe pain) & $6(15 \%)$ \\
\hline Postoperative nausea & $2(5 \%)$ \\
\hline
\end{tabular}

